\title{
Exploring prospective teachers' noticing of students' understanding through micro-case videos
}

\author{
Fadime Ulusoy $^{1}$ (D) . Erdinç Çakıroğlu ${ }^{2}$
}

(c) Springer Nature B.V. 2020

\begin{abstract}
This paper explores the nature of prospective teachers' noticing of students' understanding as they analyze and discuss middle school students' understandings of trapezoids in micro-case videos in the context of geometry. In this exploratory study, the data were obtained from eight prospective middle school mathematics teachers through individual video analysis, reflection papers, and group discussions. The results indicated that the use of purposeful micro-case video designs based on prospective teachers' background knowledge of quadrilaterals allowed them to be productive in video analyses and discussions. In individual video analyses, prospective teachers attended to various mathematical elements to identify students' responses but did not always use them to make interpretations of each student's understanding of trapezoid. In the group discussions of the micro-case videos, in contrast, prospective teachers could provide alternative interpretations of students' understanding by identifying links between the mathematical elements in students' responses and the characteristics of students' understandings. In the group discussions, they provided more detailed and specific instructional actions to support each student's understanding of trapezoid than their individual video analyses. This study suggests practical implications for teacher education programs on how to use video cases (e.g., firstly, working individually and then having group discussions about the videos) to explore prospective teachers' professional noticing skills. Considering prospective teachers' background knowledge of related mathematical contents, this study can also inspire future studies on how to design effective videos about students' mathematical understanding.
\end{abstract}

Keywords Prospective teachers · Professional noticing · Micro-case videos · Students' mathematical understanding $\cdot$ Geometry

Fadime Ulusoy

fadimebayik@gmail.com

1 Mathematics and Science Education Department, Kastamonu University, Kastamonu, Turkey

2 Mathematics and Science Education Department, Middle East Technical University, Ankara, Turkey 


\section{Introduction}

Listening to students and paying attention to their thought processes enable teachers to effectively monitor mathematical learning and to design suitable instructional actions based on students' needs (Franke et al. 2009; Jacobs et al. 2007). In recent years, teacher educators have been emphasizing the importance of teachers' skills to notice significant moments in learners' actions in order to attend, interpret, and respond to students' thinking (e.g., Jacobs et al. 2010; Sherin and van Es 2009; Star and Strickland 2008). However, identifying and interpreting mathematical details of students' understanding in a classroom environment are not easy to learn for prospective teachers (Crespo 2000; Sherin and van Es 2009). Prospective teachers' professional noticing skills do not develop naturally (Stockero et al. 2017b), but they can learn how to notice if teacher preparation programs provide opportunities for them to understand what and how students think in a mathematical situation in structured educational settings (Stockero et al. 2017a). Thus, researchers have begun to pay attention to how prospective teachers attend to and interpret particular mathematical elements in students' responses. Moreover, they are paying more attention to how they provide instructional actions on the basis of students' understanding in specific mathematical contexts such as early arithmetic reasoning (Jacobs et al. 2010; Schack et al. 2013), proportional reasoning (Fernández et al. 2013; Son 2013), derivative (Sánchez-Matamoros et al. 2019), and pattern generalization (Callejo and Zapatera 2017). In these studies, researchers mostly ask prospective teachers to reflect upon students' understandings in written or video cases. In those cases, noticing is narrowed down to particular instances of students' thinking and away from the complexity of irrelevant classroom interactions (Stockero et al. 2017a). The common idea is that the professional noticing of students' mathematical thinking is not simply about detecting what is correct or incorrect in their responses, but about reasoning out the meaning of the students' responses from both mathematical and cognitive standpoints. It is also about deciding on appropriate instructional actions on the basis of students' understandings.

In addition to written cases, video cases have been frequently used by the researchers in order to explore and support prospective teachers' noticing of students' thinking in recent years (Mitchell and Marin 2015; Schack et al. 2013). In video-based interventions, researchers generally use classroom videos that include information about multiple dimensions of a classroom environment such as the students, the teacher, management, climate, pedagogy, and mathematical thinking (e.g., Sherin et al. 2011; van Es and Sherin 2008). Although analyzing and discussing classroom video cases help prospective teachers to improve their noticing skills (Santagata et al. 2007; Schack et al. 2013), they also have various difficulties in noticing critical instances in classroom videos (Peterson and Leatham 2009; Stockero et al. 2017b; Superfine et al. 2015). Due to the complex nature of classroom environments, students' mathematical thinking is not always clearly distinguishable in the video cases (Freese 2006; Mitchell and Marin 2015). When such cases are used in teacher education, teachers tend to notice instances that are less relevant to students' mathematical thinking such as classroom management or climate, especially in early video sessions (Santagata et al. 2007; Sherin et al. 2011; Star and Strickland 2008). Thus, noteworthy instances related to student thinking may go unnoticed in the analysis of raw classroom videos (Peterson and Leatham 2009; Stockero and Van Zoest 2013). Moreover, the presentation of a raw video to prospective teachers is not usually effective without making certain edits (Seago et al. 2018; Ulusoy 2020). 
Considering the limitations of classroom video cases, we believe that the production and use of micro-case video clips (MCVCs) can be an effective alternative approach to foster prospective teachers' professional noticing skills. Micro-case video clips are educational video cases that involve a collection of specifically selected and edited cases related to an individual learner's thinking process on a particular content-related task or a problem situation in a non-classroom environment (Ulusoy 2020; Ulusoy and Çakıroğlu 2018). Evidence from prior studies suggests that micro-case videos functioned as a catalyst for prospective teachers to improve their noticing skills of students' thinking in their one-onone mathematical interactions with students at schools (Ulusoy and Çakıroğlu 2018). In this study, we used micro-case videos to explore professional noticing skills of prospective middle school mathematics teachers in the context of geometry, more specifically in trapezoids.

The current study contributes to research on teacher noticing in three important ways. Firstly, our reason for focusing on trapezoids is that according to the previous research, this concept leads to several alternative understandings for both teachers and students (e.g., Fujita 2012; Fujita and Jones 2007; Leung 2008; Popovic 2012; Ulusoy 2015). In this sense, this concept has the potential to yield a rich discussion with prospective teachers on both students' and their own understanding. Thus, our study extends the previous research about context-specific noticing because prospective teachers' professional noticing has not previously been studied in the geometry context by using video cases. Secondly, we designed and used micro-case videos that involved students' unexpected and inconsistent responses about trapezoids that prospective teachers could not anticipate in the pre-interviews. Kang and van Es (2019) think that "identifying the right video is challenging because what is "right" largely depends on situations and preservice teachers" (p. 7). At this point, we think that this study has practical implications. Exploring the design and use of micro-case videos can help to improve prospective teachers' noticing of students' mathematical understanding (Ulusoy 2020). Thirdly, our study includes a different methodological approach, in which individual video analyses are followed by group discussions about the MCVCs. Yet, in most of the noticing studies, researchers ask prospective teachers to write reflections on either students' written solutions or students' responses in the videos. Therefore, we think that this study can also provide great methodological contributions to the noticing literature. Specifically, the following research questions guided this study: (1) How do prospective middle school mathematics teachers attend to and interpret students' understanding of trapezoid in terms of mathematical elements during and after video sessions? (2) What instructional actions do prospective teachers propose during and after video sessions based on students' understandings of trapezoids?

\section{Noticing within and among}

Noticing in professional or intentional meaning is different from everyday noticing (Mason 2002) since learners realize complex situations in particular ways in professional noticing (Jacobs et al. 2010). For example, van Es and Sherin (2002) offered three key aspects of teacher noticing: "(1) identifying what is important or noteworthy in a classroom situation, (2) making connections between the specific events and the broader principles of teaching and learning, and (3) using what one knows in the context to reason about classroom interactions" (van Es and Sherin 2002, p. 573). Researchers have proposed different perspectives about what is involved in teacher noticing and how to measure and improve it, but they agree that noticing involves two common features: attending to particular noteworthy 
events and making sense of them. More specifically, Stockero et al. (2017a) have proposed a categorization for noticing studies such as (a) noticing among instances and (b) noticing within an instance. The first category is related to studies on noticing among instances. In such studies, teachers (or prospective teachers) select the instances they see important in a classroom video. Then, they explain and discuss the reasons why they think those particular instances are noteworthy in a video club design (e.g., Sherin and van Es 2009; van Es 2011). For example, Sherin (2007) identified how the teacher makes decision about where to pay attention in a given moment because classroom context is very complex. In this sense, she grouped selective attention into two main categories as actor (e.g., teacher, student, and other) and topic (e.g., management, climate, pedagogy, and mathematical thinking). Likewise, Star and Strickland (2008) identified five categories of noteworthy instances in a classroom video such as classroom environment, tasks, management, mathematical content, and communication.

It is useful to notice different instances in the classroom; however, it might be difficult to pay attention to every instance in a lesson, especially for prospective teachers (Leatham et al. 2015). For this reason, researchers have begun to focus on noticing within an instance. In such studies, (prospective) teachers are asked to analyze a specific instance of student thinking. As a prominent example of noticing within an instance, Jacobs et al. (2010) focused on "professional noticing of children's mathematical thinking" in the context of whole-number operations. In a cross-sectional study, they collected data from both prospective elementary school teachers and experienced K-3 teachers whose experienced differed in length by using two video clips involving children's strategies in problem solving processes. They provided three interrelated skills for professional noticing of children's mathematical thinking which are "(1) attending to children's strategies, (2) interpreting children's understandings and (3) deciding how to respond on the basis of children's understandings" (p. 173). The first skill is related to how teachers attend to mathematically significant events in the details of strategies that children use while solving problems. Second skill refers to how teachers construct an understanding of children's mathematical thinking. The final skill is mainly related to teachers' ways of reasoning when responding to children's mathematical understandings. Their results revealed that teachers had difficulties in all three interrelated skills of professional noticing of children's mathematical thinking. They concluded that teachers' professional noticing is a complex and challenging issue similar to the complexity of students' ideas. Based on Jacobs et al.'s study, Schack et al. (2013) examined the development of prospective teachers' professional noticing skills in early numeracy. They administered a pre- and post-assessment to measure the three interrelated skills after prospective teachers watched a short video clip that included a child's strategies in arithmetic topic. Their findings suggest that prospective teachers are better at explaining the details of the strategies that children use than interpreting them as they see them in the video.

In addition to noticing studies on early arithmetic reasoning, mathematics educators have begun to pay attention to prospective teachers' professional noticing skills in other specific mathematical contents (e.g., proportional reasoning (Fernández et al. 2013; Son 2013); derivative (Sánchez-Matamoros et al. 2019); pattern generalization (Callejo and Zapatera 2017)). For example, Fernández et al. (2013) characterized 39 prospective primary school teachers' noticing of students' mathematical thinking when they examined six students' solutions to proportional and non-proportional problems. They found that most prospective teachers could not distinguish proportional problems from additive problems. They found that only three prospective teachers distinguished proportional problems from additive problems by identifying mathematical elements and students' profiles. In 
secondary school context, Sánchez-Matamoros et al. (2019) aimed to explore prospective secondary school teachers' professional noticing skills regarding the concept of derivative and the relationships between them. They concluded that deciding how to respond on the basis of students' mathematical thinking is the most difficult skill considering the majority of prospective teachers' procedural actions. In this study, we adopted noticing within an instance of students' understanding of trapezoids by using MCVCs. We think that this study can provide contributions to the research on teacher noticing in the geometry context. When reviewing the literature, we recognized that researchers used the term student's thinking in context-focused noticing studies that include students' problem solving process (Fernández et al. 2013; Jacobs et al. 2010). Besides, in the studies of quadrilaterals that include defining, drawing, and classification of quadrilaterals, researchers prefer to use student's understanding instead of student's thinking (Fujita 2012; Fujita and Jones 2007). In parallel with previous studies and the nature of tasks used in this study, we decided to use noticing of student's understanding.

\section{Characteristics of students' understanding of quadrilaterals}

Comprehension of the attributes and properties of quadrilaterals is crucial to construct the inclusive relationship between quadrilaterals (e.g., every square is a rectangle). The inclusive relation of quadrilaterals has contributions to the development of geometrical thinking, mathematical argumentation and proof (Fujita 2012; Fujita and Jones 2007). Among quadrilaterals, a trapezoid has two different definitions in geometry textbooks: (a) exclusive definition and (b) inclusive definition (Usiskin and Griffin 2008). While "a quadrilateral with exactly one pair of opposite parallel sides" is the exclusive definition of trapezoid, "a quadrilateral with at least one pair of opposite parallel sides" is the inclusive definition. Both definitions have strengths and drawbacks (Josefsson 2013). For example, the exclusive definition is useful when students are first shown the special types of quadrilaterals. According to inclusive definition, parallelogram, rhombus, rectangle and square are classified as trapezoid. This is the main strength of the inclusive definition because a property proved as a trapezoid automatically holds for other quadrilaterals with two pairs of opposite parallel sides. Therefore, the choice of the definition of trapezoid is crucial for understanding the nature of inclusive relations of quadrilaterals (see Fig. 1).

Tall and Vinner (1981, p. 152) define concept definition as "a form of words used to specify that concept" and concept image as "the total cognitive structure that is associated
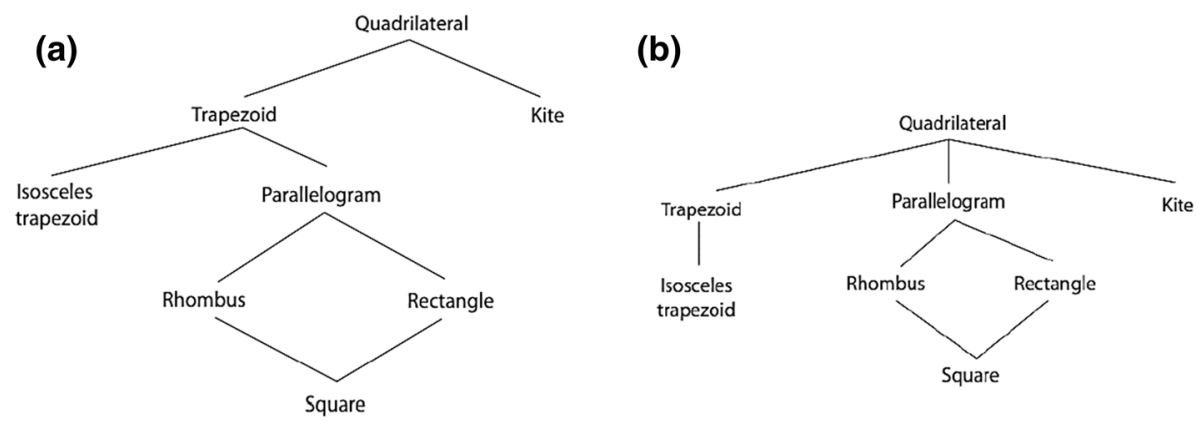

Fig. 1 Hierarchy of quadrilaterals based on a inclusive definition and $\mathbf{b}$ exclusive definition of trapezoid (Popovic 2012, p. 198) 
with the concept, which includes all the mental pictures and associated properties and process." Vinner (1991) thinks suitable and robust interactions between concept definition and concept image might guarantee the conceptual learning. Unfortunately, learners do not make sense of the link concept image and concept definition in a strong and accurate way because there might be irrelevant properties about the concept arousing in students' mind. For instance, students have an inflexible mental image of some shapes (De Villiers 1994), and they think that "a square is not a parallelogram because parallelograms are slanted" or "a square is not a trapezoid because square has equal length sides." Consequently, contradictions between concept image and concept definition emerge, which may result in misconceptions in classification of quadrilaterals. For example, students who mostly experienced prototypical examples of trapezoid (e.g., right/isosceles trapezoid) do not accept parallelogram, rhombus or rectangle as an example of trapezoid even if they know the inclusive definition of trapezoid verbally (Ulusoy 2015). Therefore, students are not at the expected level of understanding geometric concepts due to the risk of overusing prototypical examples (Fujita 2012). Moreover, some middle school students overgeneralized and accepted a quadrilateral with no parallel opposite sides or a polygon with more than four sides as trapezoid by ignoring the critical attributes of the concept of trapezoid (Ulusoy 2015). In this regard, Popovic (2012) thinks that students can be asked to write a personal definition of trapezoid after examining trapezoids and non-trapezoids, which prevents them from focusing on visual properties of prototypical shapes. Furthermore, she suggests that such activities can help students notice the arbitraries of definitions by actively participating in the process of defining and classifying quadrilaterals.

Not only students but also prospective teachers and in-service teachers have difficulties in defining and classifying quadrilaterals (Butuner and Filiz 2017; Fujita and Jones 2007; Okazaki and Fujita 2007). For instance, Türnüklü et al. (2013) found that prospective teachers had incomplete connections between concept image and concept definition and had difficulties in drawing trapezoids. As a result, they could not establish hierarchical relation between trapezoids and parallelograms. The characteristics of students' understandings of quadrilaterals are relevant for prospective teachers because interpretations of students' understanding of geometric shapes involve interpreting details of mathematical elements, which are related to concept image/definition, prototypical images and hierarchical relations of quadrilaterals. Thus, trapezoid is a difficult concept for both students and prospective teachers, and it requires to know critical mathematical elements.

\section{Method}

An exploratory study was used to examine how prospective teachers notice students' understanding in MCVCs as they engaged in video analysis and discussion sessions. This method provided an in-depth analysis of how prospective teachers think and reason with respect to professional noticing skills of attending, interpreting, and responding to students' understanding in micro-case videos in the context of geometry.

\section{Participants}

As the participants of the study, we selected fourth-year (senior) prospective teachers in an undergraduate teacher education program at a state university in Turkey. Prospective teachers who graduate from this program are qualified to be mathematics teachers in middle 
schools from grade 5 to grade 8 (age 11-14). We purposively focused on those prospective teachers because we expected that they have the highest potential of obtaining deep information from on pedagogical content knowledge considering the nature of the courses in the teacher education program. This study was carried out with eight prospective mathematics teachers (PMTs). We also used maximum variation sampling in terms of their CGPAs (cumulative graduate point average). Emel and Maya had CGPAs between 3.50 and 4.00. Beril, Oya, Zehra, and Ece had CGPAs between 3.00 and 3.50, and Aslı and Deniz had CGPAs between 2.00 and 2.50. By this sampling way, we aimed not to examine the relationship between participants' academic achievement and noticing skills. Instead, we used such a participant selection way to enrich the productivity of group discussions.

\section{Production of micro-case videos}

Firstly, we made classroom observations to get information about students' characteristics, achievement levels, and talkativeness. Then, to produce micro-case videos, we conducted case production interviews with 16 volunteer seventh-grade students at a middle school located in Ankara, Turkey. In the interviews, we asked various questions about quadrilaterals such as their definition, drawings, identifications, properties, and hierarchical relations. Clinical interviews enabled the researchers to enter students' mind to examine individual differences and their mathematical understandings (Clement 2000; Hazzan and Zazkis 1999). In order to videotape students' understandings, we utilized single-camera video production technique (Musburger and Ogden 2014). Hence, we obtained 1000 min of raw video data. However, raw videos involve many useful and useless events which reduce the productivity of group discussions in teacher education (Sherin et al. 2009; Superfine et al. 2017). Therefore, it is necessary to find a reasonable and careful way of preparing video segments to develop prospective teachers' noticing skills. In this sense, Talanquer et al. (2015) found that examining students' inconsistent responses improves teachers' noticing skills. Some researchers also think that the selection of significant events is actually related to viewers' background knowledge (Talanquer et al. 2015). In a recent study, Glogger-Frey et al. (2018) evaluate prospective teachers' prior knowledge as a prerequisite to learn how they assess students' learning strategies. Based on these studies, in order to prepare and edit MCVCs, prospective teachers' prior knowledge of quadrilaterals was also examined. In this regard, we conducted a pre-interview with each prospective teacher to gain insights into their knowledge of trapezoids. In the interviews, we asked them to write personal and instructional definitions of trapezoid, to draw at least five different examples of trapezoid and to identify trapezoids among various quadrilaterals and polygons. Then, we asked them how students can define, draw and identify trapezoids. Pre-interview data were examined to classify PMTs' inadequacies/difficulties about trapezoids and their anticipations about students' understandings. Pre-interviews indicated that PMTs had difficulty anticipating students' inconsistent responses and errors of overgeneralization and underspecification. Thus, we decided to select MCVCs including students' inconsistent responses and different types of errors (see Table 1).

The length of the educational video is crucial for increasing the efficiency of video analysis and discussions (Jaworski 1990; Seago 2004). In most of video-based studies, the duration of video content is usually less than $10 \mathrm{~min}$ (e.g., Colestock and Sherin 2009; van Es et al. 2017). Accordingly, we aimed MCVCs not to exceed $10 \mathrm{~min}$. Moreover, since PMTs are inexperienced in recognizing student's understandings, it is difficult for them to deepen students' thoughts in the presence of intensive noisy composition of a video 
Table 1 Information about MCVCs

\begin{tabular}{ll}
\hline Videos & Students' \\
\hline MCVC1 \\
4.54 min
\end{tabular}

(a)

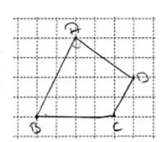

(b)

Fig. 2 a Student-1's five-sided drawing for trapezoid, b second trapezoid drawing

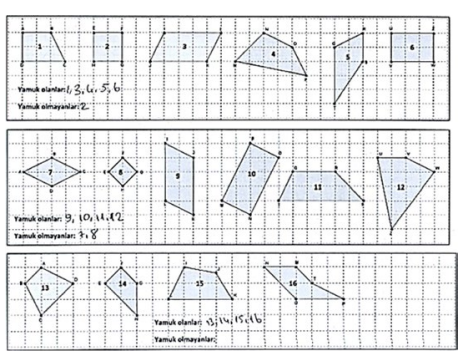

Student-1 described trapezoid as "an irregular polygon that has non-equal length sides." She initially drew a five-sided polygon as an example of a trapezoid (see Fig. 2a) although she said that she could not remember how to draw a trapezoid. Later in the clip, although the student stated that trapezoids had no parallel sides, she constructed an additional example of a trapezoid as an ABCD quadrilateral with parallel opposite sides of [AB] and [DC] on the grid paper (see Fig. 2b). When the researcher asked if trapezoids had any parallel sides, the student said that trapezoids (in Fig. 2b) had no parallel sides. In the identification task, the student selected 1-3-5-6-9-10-11-12-13-14-15-16 as trapezoids

MCVC2 $10.05 \mathrm{~min}$

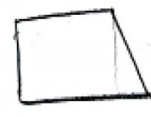

(a)

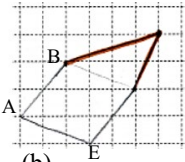

(b)

Fig. 3 Student-2's a first and b second trapezoid drawing

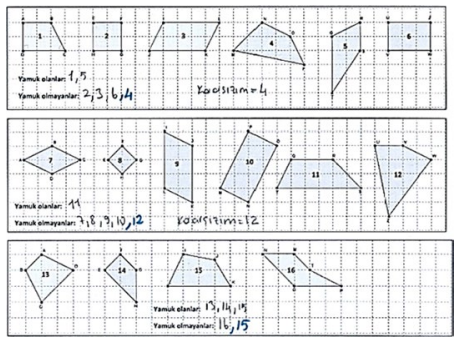

Student-2 described trapezoid as "a shape formed by putting a triangle next to a square or rectangle side by side" and drew the trapezoid in Fig. 3a as an example of a trapezoid. The researcher asked her to complete the partial drawing of a trapezoid in Fig. 3b in which only the sides of $[\mathrm{AB}]$ and $[\mathrm{AE}]$ were given. The student constructed a five-sided shape instead of a quadrilateral although she claimed that trapezoids had four sides. In the identification task, the student identified 1-5-11-13-14-15 as examples of trapezoids. The student said that she was not sure about 4 and 12 due to their positions and that those shapes might be trapezoids. In the interview, she changed her decisions after she remembered the parallelism of opposite sides in trapezoids and only selected 1-5-11-13-14 as trapezoids

(Superfine et al. 2017). For this reason, we deleted some noisy parts such as nonmathematical or non-pedagogical aspects of videos (e.g., blurred scenes; empty scenes where student's paper is not visible; long periods of time that the student waits unresponsively or spends drawing).

\section{Characteristics of students' understandings in micro-case videos}

Responses of two students to the tasks were prepared based on previous studies on students' identifications of trapezoids (Ulusoy 2015, 2016). These MCVCs mainly include noteworthy mathematical elements where the students had various difficulties and 
inconsistencies on the tasks or changed their responses during the videos. Students' written responses might have limitations of showing such mathematical details. However, MCVCs enable the researchers to see students' understandings within a process. Table 2 presents characteristics of students' responses in terms of mathematical elements.

\section{Data collection and analysis}

At the beginning, PMTs analyze MCVC1 individually in their personal laptops. Then, they wrote a reflection paper in relation to student-1's responses to trapezoid tasks. In the reflection paper, they wrote what they noticed about student's understanding of trapezoids and how they interpreted the student's understanding of trapezoids. They also provided instructional strategies considering the student's understanding of trapezoids. After PMTs completed to write the reflection papers, we initiated a group discussion. In the group discussion, we used a framework for the facilitation of PMTs' analysis of video (van Es et al. 2014). Based on the framework, we had the roles of (1) orienting the group to the video (e.g., What did you notice in the video? What did you find interesting about student's understanding of trapezoid?), (2) sustaining an inquiry stance (e.g., Can you tell me more about why the student defined trapezoids in such a way? I understood your idea that suggests that student has a misunderstanding, but can you give some details what you exactly mean?), (3) maintaining a focus on the video (e.g., Is there any different idea about the possible reasons of student's incorrect drawing? What would your instructional actions be if your students made the same mistakes?), and (iv) supporting group collaboration (e.g., You said that the student combined a triangle and a square to make a trapezoid. That is interesting. It seems reasonable, but could there be another interpretation?). In group discussions, PMTs were able to watch the video segments that they wanted to remember again. At the end of individual video analysis and group discussion of MCVC1, we followed a similar procedure for MCVC2. Then, we requested PMTs to write a post-discussion reflection paper (PDRP). In these reflection papers, we asked them to write how they influenced their peers' ideas in terms of noticing mathematical elements in the student's understanding of trapezoid and to propose specific instructional actions to help students understand trapezoids in the videos.

For data analysis, we examined reflection papers of individual video analysis, group discussions and post-discussion reflection papers. We mainly analyzed the data through qualitative methods (Strauss and Corbin 1998). First, we made a chronological order for video sessions that consisted of PMTs' individual video analysis reflections, group discussions and post-discussion reflections. Next, we took specific field notes about PMTs' professional noticing of students' understanding of trapezoid. We began to identify and divide the data set into idea units in order to code PMTs' written statements and verbal explanations. Idea unit is defined as "a distinct shift in focus or change in topic" (Jacobs et al. 1997, p. 13). In this regard, any idea unit in a reflection paper can involve one statement or a paragraph consisting of more than one statement or a conversation on a mathematical issue. Consequently, the length of an idea unit generally depended on the content (e.g., definition, drawing, and identification of trapezoids) and the types of data source (e.g., reflection paper and group discussions). Hence, the two researchers divided the data to determine the unit ideas. Interrater reliability was $92 \%$ for individual reflection papers and $94 \%$ for group discussions. Disagreements were resolved through discussion. Finally, we focused on how PMTs noticed students' understandings of trapezoids during and after video sessions. In this sense, we noted how each PMT attended to, interpreted, and decided how to respond 
Table 2 Characteristics of students' responses in MCVCs

\begin{tabular}{ll}
\hline Videos & Characteristics \\
\hline MCVC1 & Student-1 describes trapezoid as an irregular polygon \\
having non-equal length sides. Based on this \\
definition, the student draws a five-sided polygon \\
to illustrate trapezoid (see Fig. 2a). This shows that \\
student's concept image of a trapezoid is formed \\
based on the meaning of the word trapezoid in the \\
colloquial language, which means uneven or disor- \\
ganized. Furthermore, the student treats non-exam- \\
ples as examples of trapezoids in the identification \\
task without considering the formal definition \\
and critical attributes of trapezoids. The student \\
states that trapezoids have no parallel sides, but \\
constructs a quadrilateral that has opposite parallel \\
sides (see Fig. 2b). Besides, the student selects all \\
parallelograms and rectangles as trapezoids, which \\
reveals student's insufficient knowledge of parallel- \\
ism. Overall, student's responses reflect errors of \\
overgeneralization and inconsistencies in definition, \\
drawings, and identification of trapezoids \\
Student-2 firstly defines and draws a trapezoid based \\
on the concept image of a right trapezoid. The \\
student has difficulties in drawing a trapezoid in \\
non-prototypical position on the grid paper by \\
ensuring at least one critical property of trapezoids \\
(e.g., parallel opposite sides) due to the influence of \\
prototypical concept images. Identification task also \\
indicates student's ambiguity concerning critical \\
properties of trapezoids. The student tries to select \\
trapezoids based on exclusive relations of quadrilat- \\
erals although she defines trapezoids inclusively. As \\
a result, the student does not select parallelograms, \\
rhombus, rectangles, and squares as trapezoids, \\
which reflects an error of underspecification. The \\
changes in the student's responses in the identifica- \\
tion task show how the student makes an effort \\
to identify trapezoids considering the critical \\
properties rather than the position and appearance \\
of shapes after examining the identification task. \\
Finally, the student identifies trapezoids according \\
to exclusive relations of quadrilaterals
\end{tabular}

on the basis of students' understanding of trapezoids in the individual video analysis. We prepared codes according to the studies that investigate prospective teachers' professional noticing skills in specific mathematics domains (e.g., Jacobs et al. 2010; Sánchez-Matamoros et al. 2019). Table 3 shows a summary of the coding of professional noticing skills.

In coding of how PMTs attended to mathematical details in students' understandings of trapezoids, we identified mathematically significant details (see Table 2). For instance, if PMTs unpacked the most of mathematically important details in the student's responses providing a detailed description, we coded that PMTs' comments provided robust evidence. In that process, we did not require PMTs to grasp all mathematical details in students' responses "because demonstrating expertise does not require individuals to always recall and understand everything; even teachers who have acquired expertise in attending to children's strategies can lose focus" (Jacobs et al. 2010, p. 179). However, if PMTs identified some mathematically significant details in students' responses rather than identifying most 
Table 3 Coding scheme

\begin{tabular}{ll}
\hline Skills & Explanation \\
\hline Attending to mathematical elements & $\begin{array}{c}\text { It includes the descriptions related to mathematically significant } \\
\text { details like how students define/draw/identify trapezoids and the } \\
\text { consistencies or inconsistencies in students' mathematical actions } \\
\text { in defining/drawing/identifying trapezoids }\end{array}$ \\
Interpreting students' understandings & $\begin{array}{c}\text { It includes comments that refer to the practice of making sense of } \\
\text { a student's mathematical ideas like what students understand. } \\
\text { It also refers to the reasons that underlie students' understand- } \\
\text { ings of trapezoids in a variety of ways on the basis of students' } \\
\text { responses and the research on quadrilaterals (e.g., prototypicality, } \\
\text { concept image/concept definition, inclusive/exclusive relations of } \\
\text { quadrilaterals) } \\
\text { It includes instructional actions proposed based on each student's } \\
\text { understanding of trapezoids. It provides a rationale for selected } \\
\text { instructional actions considering the student's specific under- } \\
\text { standings of trapezoids }\end{array}$ \\
$\begin{array}{c}\text { Deciding how to respond based on } \\
\text { students' understandings }\end{array}$ & \\
\hline
\end{tabular}

Specific examples were explained in the results section

of the mathematical elements, we coded that PMTs' comments provided medium evidence. Responses demonstrating medium evidence of attending to students' understanding of trapezoids can be phrased in many ways, but they occasionally express context-specific language and details about students' understanding in terms of concept images/concept definition, prototypicality, and hierarchical relations of quadrilaterals in identifications. If PMTs used a few mathematical elements to describe students' responses and only provide general descriptions of students' answers, we coded that they provided low evidence to describe mathematical elements in students' responses. In such comments, PMTs tend to mention if the student defines, draws, or identifies trapezoids successfully or not in a chronological order, but they avoid explaining how s/he does them mathematically.

We coded PMTs' interpretations of students' understanding of trapezoids in three groups (1) robust evidence, (2) medium evidence, and (3) low or no evidence. If a prospective teacher made sense of the mathematically relevant details in the student's understanding and notes that included what the student understood about trapezoids, we coded that this prospective teacher interpreted students' understanding of trapezoid providing robust evidences. This was completely consistent with both student's understanding and the literature about quadrilaterals regarding concept images/concept definition, prototypicality and classification of quadrilaterals. However, if PMTs commented on the students' understandings in broad terms, we coded them as containing medium evidence. Although such comments include conclusions and specific connections to the students' understanding of trapezoids, they are generally limited and overgeneralized (e.g., student understood trapezoids in MCVC2, but the student did not know about trapezoids in MCVC1). Finally, if responses did not provide any evidence of interpretation of students' understanding or provide a general perspective on what they learn about mathematics teaching and learning from videos, we coded them in the group of low or no evidence.

To examine PMTs' instructional actions, we used three specific categories that emerged from data: (1) general (e.g., explaining trapezoid again) (2) specific-undetailed (e.g., for student-2, using geoboard is useful), and (3) specific-detailed (e.g., they include information about which student the instructional action is proposed for, and how and why it is 
used). We grouped PMTs' instructional actions as individual video analysis and post-discussion reflection papers. Specific examples of these categories are given in the result section. After the analysis of individual video analysis reflection, we also examined PMTs' explanations in group discussions, and we highlighted whether they showed changes in terms of professional noticing of students' understanding of trapezoids. At this point, the themes were extracted from classroom discussions and PDRP through content analysis (Strauss and Corbin 1998).

\section{Results}

In this section, we report to what extent PMTs attended to mathematical elements in students' understandings of trapezoids, interpreted students' understandings and provided instructional actions in individual video analysis and group discussions.

\section{Prospective teachers' professional noticing in individual video analysis}

\section{Attending to the mathematical elements in students' understandings}

For individual video analysis process, we grouped PMTs' responses into three categories considering to what extent they attended to mathematical elements to describe the students' responses: low evidence, medium evidence, and robust evidence (Table 4). PMTs' explanations indicated that Zehra used only a few mathematical elements in MCVC1 (low evidence). Table 5 shows that she realized that the student was completing a shape to get a trapezoid on the grid paper, but she did not provide details about how the student drew the trapezoid by referring to student's concept image and definition of trapezoid that is formed based on the meaning in the colloquial language. Additionally, she missed out all the important mathematical details in defining and identifying tasks such as overgeneralized concept definition and inconsistencies between student's identifications and definition in the video.

Compared to the responses including low evidence of attending, three PMTs focused on more mathematical elements to describe students' responses in both MCVCs (medium evidence). They generally noticed and distinguished students' concept images/definitions

Table 4 Noticing mathematical elements in students' responses

\begin{tabular}{llllll}
\hline PMTs & MCVC1 & & & MCVC2 & \\
\cline { 2 - 3 } \cline { 5 - 6 } & Attending & Interpreting & & Attending & Interpreting \\
\hline Oya & Robust & Robust & & Robust & Robust \\
Zehra & Low & Low & & Medium & Low \\
Maya & Medium & Medium & & Robust & Medium \\
Asli & Medium & Medium & & Medium & Medium \\
Emel & Robust & Medium & & Robust & Robust \\
Ece & Robust & Robust & & Robust & Robust \\
Deniz & Medium & Low & & Medium & Medium \\
Beril & Robust & Robust & Robust & Robust \\
\hline
\end{tabular}


Table 5 Representative examples of PMTs' attending responses

Representative examples in attending

Low evidence

This student both surprised and did not surprise me. Although there was parallelism in her trapezoid drawing, the student said there were no parallel sides. Correct and incorrect answers are given by chance.

There is almost nothing about the mathematical concept in the student's mind [Zehra-MCVC1 reflections]

Medium evidence

The student's knowledge of trapezoid is weak. Interestingly, she described trapezoids according to colloquial language... I did not expect the student to draw such a shape for a trapezoid...There is inconsistency between the student's understanding of parallelism and her selections of trapezoids [Maya-MCVC1 reflections]

Unsurprisingly, Student-2 gave the definition based on a typical trapezoid shape. However, I am surprised to see student-2's selections of trapezoids. The student's selections are different from her definition. She used visual properties of a typical shape [Zehra-MCVC2 reflections]

Robust evidence

The student defined a trapezoid based on the daily use of "trapezoid" in Turkish. Synonym words create problems. According to this student, a shape having non-equal sides is called a trapezoid. Based on this definition, the student drew a five-sided polygon. It is very interesting... She did not know inclusive relations of quadrilaterals. She did not include square and rhombus in the trapezoid group, but it is consistent with the student's misperception of a trapezoid, which is an irregular polygon. Although the [AB] was parallel to $[\mathrm{DC}]$ in the student's shape, the student said there was no parallelism in the shape. She did not know what parallelism meant [Emel-MCVC1 reflections]

formed by prototypical shapes or linguistics factors. However, they were mostly not able to attend to the most of mathematical elements in students' understandings related to drawings or identifications. For example, Maya attended to the student's definition that is formed based on the meaning in the colloquial language. She found the student's drawings interesting but did not provide the details of what and how the student drew to illustrate the trapezoid in MCVC1 (see Table 5). Similarly, Zehra attended to the role of prototypical images in the student's definition in MCVC2. Although she noticed an important mathematical element in the student's definition, she attended to the student's identification of trapezoids with general descriptions. However, she did not attend to prototypicality in student's drawings of trapezoids and exclusive relations of quadrilaterals in student's identifications in MCVC2.

Finally, four PMTs provided robust evidence of attending to the student's understandings in MCVC1. That number rose to five in MCVC2 since Maya increased her attending by providing robust evidence in the reflection of the second video. PMTs' descriptions included most of the mathematically important elements related to how the student used the grid paper to draw a trapezoid, completed a shape to form a trapezoid and defined and identified trapezoids and non-trapezoids. When they described students' responses, they commonly used crucial mathematical terms regarding conceptualizations of quadrilaterals. As a representative example (Table 5), being aware of the role of linguistic factors in the student's errors of overgeneralization in MCVC1, Emel described student's definition, drawings, and identification of trapezoids. Furthermore, she described the nature of relations of quadrilaterals in the student's responses and established a mathematical link between student's concept image and concept definition rather than using broad and vague statements. Thus, these prospective teachers employed context-specific language when describing mathematical elements like concept image/definition formed by language or prototypical shapes and the nature of identifications of trapezoid (e.g., exclusive/inclusive classification of quadrilaterals). In addition, they mentioned the inconsistencies in students' definition, drawing, and identification processes in terms of mathematical elements. 
Table 6 Representative examples of PMTs' interpreting responses

Representative examples in interpreting

Low evidence

Student-1 doesn't understand what trapezoid means. She heard something in the class and used these things in her definition and identification...[Zehra-MCVC1 reflections]

Medium evidence

Student's drawing indicates that she knows the meaning of a trapezoid as in the colloquial language. The student had various difficulties in identifications of trapezoids because she focused on the length of sides in polygons. The student had critical problems with definition of trapezoids [Asl1-MCVC1 reflections]

The student visualized trapezoids as the combination of a triangle and a square. This definition does not include other trapezoids. I think their teacher gives similar examples. The student changed her ideas about parallelism in the video. This indicates that she does not fully know the meaning of a trapezoid [Asl1MCVC2 reflections]

Robust evidence

Student-1 does not remember trapezoids. Instead, she defines and visualizes the shape considering the meaning of trapezoids in the colloquial language. For this reason, she does not consider a trapezoid as a four-sided polygon because she drew a five-sided polygon. She focused only on non-equal sides in the shapes. Parallel with this idea, she selected all the quadrilaterals except the squares and rhombus as trapezoids [in the identification task]. Another important point is that the student said there were no parallel sides in a trapezoid, but she drew a quadrilateral with a pair of parallel sides. The reason might be that she either does not see parallelism on the grid paper, or she visualizes parallelism in a [proto]typical position $(\longrightarrow$ [Oya-MCVC1 reflections]

The student just informally described and drew the right trapezoid that she imagined because she only focused on the visual characteristics of the right trapezoid. This might be related to their teachers' overuse of typical examples of trapezoids. Hence, the student could not consider conceptual properties that are valid for all trapezoids. For this reason, the student imagines the right trapezoid when someone asks to draw an example of a trapezoid. Similar situation was observed in the student's identifications because the student identified trapezoids through typical shapes presented in the textbooks. She did not consider parallelograms and rhombus as examples of trapezoids due to her typical image of trapezoids [Emel-MCVC2 reflections]

\section{Interpreting students' understandings}

We also categorized PMTs' interpretations into three groups. Table 4 shows that two PMTs interpreted students' understanding by providing low evidence in MCVC1. For instance, Zehra mentioned whether the student knew trapezoids or not without mentioning the role of linguistic factors in students' errors of overgeneralization (see Table 6). Furthermore, she did not question possible reasons underlying the student's incorrect definition of a trapezoid and her five-sided polygon drawing. Similarly, Deniz explained whether the student knew about trapezoids in broad statements in MCVC1. Besides, three PMTs provided responses demonstrating medium evidence of interpretations of students' understandings in MCVC1. Similarly, four PMTs provided medium evidence in their interpretations in MCVC2. These PMTs interpreted students' understanding beyond the expressions like students knew/did not know about trapezoids. However, they sometimes provided their interpretations in broad terms or provided overgeneralized conclusions about some mathematical points in the videos. More specifically, they distinguished students' errors of overgeneralization or underspecification in videos. However, they unfortunately failed to make strong connections among students' errors of overgeneralization/underspecification, concept image/definition and classifications of quadrilaterals. For example, Asli noticed the role of the colloquial language in the student's understanding of trapezoids in MCVC1 (see Table 6). She mentioned the difficulties students had in the identification task, but how 
exactly the student had difficulties identifying trapezoids was unclear. Furthermore, Asl1 focused on the meaning of student's definition and drawings in MCVC2 in broad terms. She inferred that the teacher gave limited number of examples of trapezoids in MCVC2. However, this conclusion goes beyond the evidences provided in the video.

Finally, three of PMTs' responses showed robust evidence of interpretation of students' understandings in both videos (Table 4). In the second video, in addition to three PMTs, Emel also provided robust evidence. They interpreted students' understandings in several ways and found out how both students understood trapezoids in terms of mathematical elements in a variety of ways that were all consistent with students' understanding in MCVCs and the studies on quadrilaterals. For example, Oya's responses showed that she made sense of the mathematical details such as how the student understood trapezoids (Table 6). She recognized the role of the colloquial language in student's concept image and identification task and made inferences about the reasons why the student could not draw parallels on the grid paper. These comments pointed out mathematically relevant details in students' responses with context-specific language. Similarly, some PMTs made inferences about the student's definitional error in MCVC2 by complaining about overuse of prototypical examples in mathematics textbooks and teacher's preferences (see Emel's explanations in Table 6). Therefore, PMTs who provided robust evidence of interpretations made sense of details of each student's understandings in different ways.

\section{Deciding how to respond on the basis of students' understandings}

Table 7 illustrates the nature of PMTs' instructional actions in individual video analysis. PMTs had difficulties in deciding how to respond to students' understandings since they proposed only 15 instructional actions in total. Furthermore, they mostly provided instructional actions in general (lack of evidence) or specific-undetailed categories (limited evidence). For example, Asl1 wrote "I think that it is useful to explain the topic from the beginning again. Furthermore, the activities should be done" in the reflection paper. In this suggestion, Asli did not give details about how the topic can be explained to the student and what kinds of activities should be done for what purpose. Another example can be Zehra's written comments: "Explanations are important in mathematics. In my opinion, terms

Table 7 PMTs' instructional actions in individual video analysis

\begin{tabular}{lllll}
\hline PMTs & \multicolumn{2}{l}{ Instructional actions } & Total frequency $(\%)$ \\
\cline { 2 - 5 } & General & $\begin{array}{l}\text { Specific-unde- } \\
\text { tailed }\end{array}$ & Specific-detailed & \\
\hline Oya & 0 & 0 & 0 & 0 \\
Zehra & 1 & 1 & 0 & 2 \\
Maya & 1 & 1 & 0 & 2 \\
Asli & 2 & 1 & 2 & 5 \\
Emel & 0 & 1 & 0 & 1 \\
Ece & 2 & 0 & 0 & 2 \\
Deniz & 0 & 2 & 0 & 2 \\
Beril & 1 & 0 & 0 & 1 \\
Total frequency & $7(47)$ & $6(40)$ & $2(13)$ & $15(100)$ \\
$\quad(\%)$ & & & \\
\hline
\end{tabular}


should be explained well." These comments were also too general and did not include specific mathematical elements based on the student's understanding in the video. Besides, six instructional actions were specified as mathematical elements in the student's understanding of trapezoids, but they were still too broad. For example, Maya wrote "Student-2 chose shapes having visual similarity with the right trapezoid. I understood that it is necessary to show a lot of shapes to students in the teaching process." In this explanation, Maya specified student's understanding mathematically, but she did not provide details about which shapes can be demonstrated while teaching. Moreover, Aslı added a specific-undetailed instructional action considering student's difficulty with parallelism in MCVC1. She wrote "When I recognized that the student was having difficulty with parallelism, I thought that it was necessary to prepare an activity about parallelism for this student." These comments indicated that she did not mention the details of the activity. Finally, only two instructional actions were grouped as specific-detailed (robust evidence). For example, Asl1 wrote:

Student-2 focused on the right trapezoid. If I were the teacher of this student, I could give the definition of trapezoids explicitly because the student could not understand the hierarchical relationship between quadrilaterals. In addition, I will give various examples of trapezoids rather than focusing on typical examples. For this, I can use GeoGebra. In the program, I can draw a shape that can flexibly be changed according to the definition of trapezoids. By changing the sides of the shape, I can show the trapezoid to the student. Alternatively, the student cuts the paper like $\square$ to produce different examples of trapezoids. Thus, the student can understand trapezoids conceptually [Asl1, MCVC2-PDRP].

Asli's comments involved more details about how and why she decided to use instructional actions to develop student's understanding in MCVC2. In her comments, she focused on the relationship between student's concept image and concept definition of trapezoids considering the hierarchical nature of quadrilaterals with appropriate learning materials.

\section{Prospective teachers' professional noticing in group discussions}

\section{Attending to specific mathematical details in students' understandings}

Table 8 indicates how PMTs' increased their attending to students' understandings through group discussions. For example, prospective teachers who attended to many of the mathematical details in students' understandings in the individual video analysis by providing robust evidence did not add any additional explanations regarding what they attended to in the report written after the group discussion. The reason for this was that they had already figured out the important points. Only Beril who provided robust evidence in individual video analysis wrote "In the group discussion, I recognized student's non-hierarchical relations of quadrilaterals in identifications. The student recognized the rotated shapes (shapes 13-14) as a trapezoid, but could not accept parallelogram as a trapezoid in MCVC2." Thus, Beril had the opportunity to address to the specific details of the student's classification of quadrilaterals through group discussion. Furthermore, in the individual video analysis, prospective teachers who noticed some of the mathematical details in student understanding and who were grouped as the ones that presented medium evidence realized the important mathematical situations they missed out in the individual process after the group discussion. They provided robust evidence in this regard in their written explanations. As an example, Deniz, who provided medium evidence in individual analysis of MCVC1, had 





opportunities to notice details of mathematical elements regarding the colloquial language on the student's concept image at the end of the group discussion as follows:

Researcher What did you notice about [student's] understanding when you watched the video?

Maya I think she doesn't know [trapezoid concept]

Asli What a superficial definition!

Deniz When watching the video, I wondered how she found this definition

Ece She thought the meaning of trapezoid in the colloquial language

Deniz Is this because of the synonym of trapezoids in Turkish?

Beril The trapezoid concept in her mind corresponds to the irregular and uneven shape used in Turkish. She thinks that trapezoid cannot be a shape with equal length sides

Oya Yes, a shape having unequal sides is enough to be considered as a trapezoid for her

Asl1 She did not select square and rhombus but selected other shapes [as trapezoid]

Deniz Why did she identify parallelograms as trapezoids?

Oya Because all sides of parallelogram do not have equal length

Deniz Hmm. You are right

In group discussion, Deniz's explanation indicated she did not notice the linguistic factors in the student's understanding of trapezoids. After Ece's response, Deniz questioned synonym words in Turkish and student's understanding. In order to provide evidence, Beril showed the student the expression of "trapezoid has non-equal sides." When Deniz wondered the reason why the student thought parallelograms as trapezoids in the identification task, Oya immediately implied the non-equal length of sides in a parallelogram as the reason. By referring to the benefit of the group discussion, Deniz focused on the details of mathematical elements in student's understandings in PDRP. She wrote the following comments.

In the group discussion, I realized that the student visualizes trapezoid as an irregular shape with unequal length of sides due to its meaning in the colloquial language. This idea affects student's identification of trapezoids. The student overgeneralized and selected all shapes having non-equal opposite sides as trapezoid [Deniz-MCVC1 PDRP-Robust evidence].

These explanations revealed that Deniz understood the details of the student's understanding and associated student's concept image with his/her choice of trapezoid. Similarly, Maya who provided medium evidence in individual video analysis noted mathematically significant details about the student's drawings and concept image of trapezoids after the group discussion.

In group discussion, I recognized the student's inconsistent responses about drawing a shape in MCVC1. Normally, the student said there were no parallel sides but drew a shape including parallel opposite sides. I also realized that the student did not visualize trapezoid as a quadrilateral because she drew a five-sided polygon [MayaMCVC1 PDRP-Robust evidence]. 


\section{Multiple interpretations on students' mathematical understandings}

In individual video analysis, some PMTs interpreted students' understanding of trapezoids with robust evidence and provided various valid interpretations. This diversity in interpretations of students' understanding created a rich discussion environment. By this way, PMTs elaborated their interpretations of mathematical details in students' understandings by focusing on possible reasons and mathematical details. Table 9 shows the nature of PMTs' interpretations before and after the group discussions.

Some illustrative examples were presented to show what and how prospective teachers interpreted students' mathematical understandings for each video clip. For example, the researcher's prompt shifted the group discussion from attending to mathematical elements to interpreting details of the student's understanding in MCVC1.

Researcher What did you expect about student's trapezoid drawings before analyzing the video?

Emel I thought the student would draw a quadrilateral without any parallel sides as a trapezoid but she did not even draw a quadrilateral as a trapezoid let alone parallel sides

Beril I found it interesting. The student drew a five-sided polygon although they learn trapezoids in the context of quadrilaterals

Oya The student inconsistently said that there were no parallel opposite sides in her drawing, but actually there were

Ece She was unaware of whether her drawing involved parallel sides or not

Asli In my opinion, she did not know about parallelism

Emel I think she knew about neither parallelism nor trapezoids

Aslı I agree. She did not know about quadrilaterals since she drew a five-sided shape. She also identified parallelograms and rectangles as trapezoids. It is a messy classification

Ece Not messy. For the student, the absence of equal length sides in a polygon is sufficient to call it a trapezoid

In the discussion, Oya directly focused on student's inconsistent responses related to parallelism of sides in a trapezoid by giving evidence from the clip. As a response, Ece suggested that the student was not sure about the parallelism of opposite sides. According to Emel, the student drew parallel lines in the shape by chance rather than consciously arranging them. At this point, Aslı elaborated her interpretations of student's mathematical understanding by making connection between the student's identification and concept image. After the group discussion, PMTs explained their own development in noticing of student's mathematical understanding considering the student's lack of knowledge in previously learned concepts. To illustrate, Asli's and Ece's written statements are given as follows:

I recognized the influence of lack of knowledge of previously learned concepts such as parallelism on student's inconsistent drawings. I also became aware that student's image caused errors in identifications because she selected every shape as a trapezoid (excluding shapes with equal length sides) [Asl1-MCVC1 PDRP-Robust evidence].

I thought that the student solely focused on the meaning of trapezoids in Turkish. However, my friends' thought differently. They claimed that the student did not know how to construct parallelism on the grid paper. I recognized that basic geometric con- 





cepts are very important (for the conceptualization of quadrilaterals) [Ece-MCVC1

PDRP-Robust evidence].

The comments revealed that both PMTs noticed the importance of basic geometrical concepts for the student's understandings of trapezoids. Asli provided robust evidences for the details of student's overgeneralized identifications of trapezoids after the group discussion while she interpreted them in general terms in the individual analysis. In addition, although Ece provided robust evidence of interpretations in the individual video analysis, she adopted different viewpoints about why the student overgeneralized trapezoids considering the student's lack of knowledge of parallelism. Thus, she had opportunities to interpret the student's understanding in several ways.

Another example is that PMTs put forward different viewpoints about student's prototypical concept image of trapezoids in individual video analysis of MCVC2. In the group discussion, the researcher invited them to explore the reasons of prototypical concept images. As a result, the group concentrated on the student's understanding again.

Researcher Why did the student define the right trapezoid instead of other types of trapezoids?

Emel She cares about the appearance [of shape]

Asli Yes, appearance

Oya She can be unfamiliar with other trapezoid types

Researcher You may be right but almost all of the students whom I have interviewed drew the right trapezoid or isosceles trapezoid

Aslı I think their teacher might have overemphasized the right trapezoid in the lessons

(The group agreed by nodding their head)

Researcher Why might their teacher have overemphasized typical trapezoids?

Oya Maybe, there are questions mostly including typical trapezoids in exams. It is easy to calculate the area of the right trapezoid. Two sides are equal in isosceles trapezoids

Maya Teacher might have wanted students to use the triangle, square and rectangle during the area and circumference calculations considering students' familiarity with them

Asli It makes sense

The common idea about the reasons why student focused on the right trapezoid was student's focus on visual characteristics rather than the conceptual properties of trapezoids. Hereon, Asli claimed that the teacher might have overemphasized the right trapezoids in the lessons. At this point, the researcher's particular question encouraged PMTs to think of some possible reasons of overemphasis of prototypical trapezoids in the instruction. Oya thought that there might be an influence of the teacher's selection of examples in the exam questions. Maya extended these ideas focusing on the fact that the calculation of perimeter and area of trapezoids might be easy in the right trapezoid. To conclude, this dialogue was important because PMTs' comments suggested mathematics teachers' limited examples and explanations as a possible reason for the student's prototypical concept image. This made very important contributions to PMTs' noticing in terms of interpreting student's understanding. They mentioned these contributions in 
their reflection papers. For instance, two PMTs elaborated their interpretations about prototypicality.

Before the group discussion, I did not think of the reason of the student's focus on the right trapezoid. I thought that the overuse of prototypical examples in the exams and textbooks might be the reasons of student's prototypical concept formation [MayaMCVC2 PDRP-Robust evidence].

I never thought about the reason of this understanding before the discussion. My friends thought that teacher can overemphasize special trapezoids and frequently use them in exams and area/circumference calculations. I extended my knowledge of them [Emel-MCVC2 PDRP-Robust evidence].

These comments were in align with the increase in depth of PMTs' interpretations of student understanding of trapezoids. They deepened their interpretations focusing on both the role of prototypical shapes in concept formation and possible reasons of overuse of prototypical shapes.

Another striking example was given to show the developments in PMTs' interpretations of mathematical elements in the student's drawings in MCVC2. The researcher posited a question to examine PMTs' reasoning about student's five-sided drawing. Hereon, PMTs reached a point where they needed to spend time thinking further to offer possible reasons.

Researcher Why did the student draw a five-sided polygon as a trapezoid?

Emel If the researcher gave only $[\mathrm{AB}]$ and $[\mathrm{AE}]$ (see Fig. 3b) in a linear position, the student might have drawn a four-sided shape as trapezoid

Maya Ok. The researcher gave half of the shape on the grid paper, and the student had difficulty drawing the remaining part of the trapezoid to complete it

Ece She visualized CDEB as a square based on her trapezoid definition. The student tried to combine a square and a triangle by looking at her personal definition in the shape completion process

Asl1 The student might have thought [DE] and [EA] as linear because they look linear

Maya I agree with you. She could not recognize nonlinearity. She wanted to create a shape as the combination of a triangle and a rectangle

Beril In that situation, the student did not carefully draw it due to lack of knowledge of using the grid paper or might not have known all critical attributes of a trapezoid

In the discussion, two different ideas emerged about the student's five-sided polygon although the student knew the number of sides of a trapezoid. The statements at the end of the dialogue can be evaluated as an indicator of the development in Asli's and Maya's noticing of students' understanding because their comments showed robust evidence different from the ones prior to the group discussion. Moreover, PMTs' reflection papers that were written after the group discussion also showed that they commented on how the student reasoned with geometric shapes or why the student drew such a shape. For example, Zehra who provided low evidence in individual analysis wrote "In the discussion, I understood that the student could not draw linearity in different positions, so she produced a fivesided shape. Giving a shape in different positions is important." These statements indicated that Zehra provided medium evidence when interpreting student's incorrect drawing. She wrote consistent explanations with the student's prototypical concept image. Because she 
Table 10 Instructional actions presented in post-discussion reflections

\begin{tabular}{lllll}
\hline PMTs & \multicolumn{2}{l}{ Instructional actions } & Total frequency (\%) \\
\cline { 2 - 4 } & General & $\begin{array}{l}\text { Specific-unde- } \\
\text { tailed }\end{array}$ & Specific-detailed & \\
\hline Oya & 0 & 1 & 3 & 4 \\
Zehra & 3 & 1 & 0 & 4 \\
Maya & 3 & 2 & 2 & 7 \\
Asli & 1 & 1 & 3 & 5 \\
Emel & 0 & 1 & 3 & 4 \\
Ece & 0 & 2 & 2 & 4 \\
Deniz & 0 & 1 & 2 & 3 \\
Beril & 0 & 0 & 3 & $34(100)$ \\
Total frequency & $7(21)$ & $9(26)$ & $18(53)$ & \\
$(\%)$ & & & & \\
\hline
\end{tabular}

did not explain the importance of non-prototypical shapes, her comments are still unclear and too general.

\section{Focusing on alternative instructional actions on the basis of students' understandings}

In group discussions, PMTs mostly concentrated on instructional actions to overcome problems in students' understandings. As a result, PMTs began to build connections between students' understanding and specific instructional actions. According to Table 10, PMTs proposed 34 instructional actions in the post-discussion reflections. 53\% of them were specific-detailed that included such detailed information as the instructional action, for which student it is proposed, for what purpose it is used, and how it is used. Besides, $26 \%$ of the comments included specific-undetailed instructional actions. In these comments, PMTs refer to the student in the video, but they wrote broad explanations. Finally, $21 \%$ of instructional actions were grouped as general since PMTs did not specify the student's understanding and did not give details about the ways of instruction.

We also assigned certain numbers to PMTs' instructional actions to compare them before and after the group discussions. We labeled them as 1 for each general comment, 2 for each specific-undetailed comment, and 3 for each specific-detailed comment in Tables 7 and 10. We then calculated the average points of each PMT's comments on responding (e.g., for Maya, we calculated $(1 \times 1+\mathbf{2} \times 1+\mathbf{3} \times 0) / 2=1.5$ as the average point before the discussion based on Table 7 . Similarly, we calculated $(\mathbf{1} \times 3+\mathbf{2} \times 2+\mathbf{3} \times 2) / 7=1.9$ as the average point after the discussion based on Table 10. Thus, Table 11 indicates the

Table 11 Comparison of the average points in PMTs' comments on responding

\begin{tabular}{|c|c|c|c|c|c|c|c|c|c|}
\hline \multirow{2}{*}{$\begin{array}{l}\text { Average points in PMTs' } \\
\text { comments on responding }\end{array}$} & \multicolumn{8}{|c|}{ Prospective teachers } & \multirow{2}{*}{$\begin{array}{l}\text { Average of } \\
\text { total points }\end{array}$} \\
\hline & Oya & Zehra & Maya & Asli & Emel & Ece & Deniz & Beril & \\
\hline Before the group discussions & 0 & 1.5 & 1.5 & 2 & 2 & 1 & 2 & 1 & 1.4 \\
\hline After the group discussions & 2.8 & 2.5 & 1.9 & 2.4 & 2.8 & 2.5 & 2.7 & 3 & 2.6 \\
\hline
\end{tabular}


Table 12 Examples of instructional actions provided in the post-discussion reflections

Instructional actions Examples of PMTs' instructional actions

General

When teaching quadrilaterals, the developmental levels in van-Hiele should be considered. Moreover, teachers should be clear in their explanations [Maya]

It can be useful to utilize a grid paper or GeoGebra when teaching quadrilaterals [Zehra].

Students can draw shapes themselves, and they can compare their drawings. They can criticize themselves, which elaborates their understandings [Asli]

Specific-undetailed Both students think about trapezoids in a different way. To improve students' understanding, I can show different examples of trapezoids [Deniz]

Student- 2 could not pay attention to linearity of line segments. In order to overcome such a problem, we can ask the students to draw shapes on a grid paper. Using a grid paper can be useful [Oya]

Specific-detailed Student-1 had problems seeing parallelism in her trapezoid drawing. I understand that basic geometric concepts are important in learning quadrilaterals. To overcome the difficulty students have, I can use a grid paper. In the paper, I will ask the student the meaning of parallelism of line segments and to draw parallels in different positions, not only in horizontal or vertical positions. Thus, we can teach the construction of parallelism by using a student-centered teaching strategy before teaching quadrilateral to overcome misconceptions about parallelism [Ece]

In the second video, the student correctly defined trapezoid. She focused on typical shapes due to visual similarity between prototypical shapes and other shapes [nonexamples]. For the student, it is enough to call a shape as a trapezoid as long as it looks like the shape of a trapezoid in a way. In my opinion, shapes should not be shown to the student in advance. Instead, definition of the trapezoid should be given to the student before giving the shapes. Then, the student can be asked to draw shapes based on the definition [Emel]

Student-1 visualizes the trapezoid based on the meaning of a word in the colloquial language like "a trapezoid needs to have irregular sides." To prevent this misconception, I can provide information about synonym words in Turkish to emphasize the differences in their meanings at the beginning of the lesson [Beril]

development in each prospective teacher's comments on responding. This table shows all PMTs increased the quality of their comments on responding in terms of students' understandings after the group discussions.

In addition, illustrative examples of instructional actions that PMTs provided after the discussion reflections are given in Table 12. For example, in general instructional actions, Zehra could not consider herself as the teacher of the student who made the mistake in the video. She only suggested some materials such as a grid paper or GeoGebra without providing any evidence related to students' understanding in the videos. Furthermore, she did not mention the reason why using these materials is useful in teaching quadrilaterals.

In an instructional action grouped into the specific-undetailed category, Oya also recommended the use of grid paper. Different from Zehra, she explained the reason why she preferred this material. Specifically, she pointed out Student-2's concept image of a trapezoid that is formed based on visual properties of a typical trapezoid. However, she did not give specific information about what kind of shapes she asks the student to draw on the grid paper. In contrast, in specific-detailed instructional actions, Ece provided all of the following details about using the grid paper: for whom (Student-1), for what purpose (to teach parallelism) and how it is used (to draw parallel line segments in different positions). In these instructional actions, PMTs generally 
focused on significant context-specific mathematical details. In this sense, they focused on non-prototypical examples, inclusive definition of quadrilaterals, the relationship between the concept image and concept definition, and the role of important prerequisite concepts such as parallelism in the students' understandings of trapezoids in the videos. Moreover, they considered and criticized which learning materials (e.g., GeoGebra-grid paper-geoboard) can be more useful and appropriate to develop students' understandings of trapezoids formally.

\section{Discussion}

The aim of this study is to explore prospective middle school mathematics teachers' skills of attending, interpreting, and responding to students' understandings of trapezoids. They are expected to do this when they analyze and discuss the responses of students that have different characteristics of understandings of trapezoids in micro-case videos. In the individual video analysis process, PMTs mostly provided medium or robust evidence of attending to students' understandings of trapezoids. In this sense, they understood most of the mathematical elements in both students' understandings by comparing and contrasting them. For example, many PMTs recognized different nature of concept definitions and concept images of students in the videos and the role of concept formation in identifications and drawings of trapezoids. However, in some studies (e.g., Jacobs et al. 2010), researchers concluded that a substantial number of prospective teachers struggle to recall the mathematically noteworthy details of children's strategies although they watch only one child's problem-solving process in the video. In the current study, one of the main reasons why PMTs got more attention can be related to the nature of the videos. The reason for that is that micro-case videos were prepared about the contents that would draw the attention of PMTs to mathematical elements in students' understandings, which was after PMTs' background knowledge of the trapezoid concept was examined through pre-interviews. By this way, incorporating micro-case videos in teacher preparation settings promotes a more focused and detailed examination of students' mathematical understanding. In terms of interpreting students' understandings, PMTs provided some evidences in various degrees from low to robust. For instance, two PMTs made general comments on students' understandings as a whole (e.g., The student does not know about trapezoid; the student has misconceptions). PMTs generally provided medium evidence when interpreting students' understandings. In this regard, they could focus only on some of the significant mathematical elements in the videos. Another important result is that providing robust evidence that they had had understood all the mathematical aspects of student understanding in the videos, prospective teachers' responses did not guarantee that they could interpret student understanding with the same robust mathematical evidence. Similar results were reached in some important studies (e.g., Sánchez-Matamoros et al. 2019).

The results of individual video analysis also revealed that PMTs had difficulty in deciding how to respond to students' mathematical understanding as in other studies (Jacobs et al. 2010; Krupa et al. 2017; Sánchez-Matamoros et al. 2019). This is because they mostly proposed general instructional actions without referring to Student-1 or Student-2 in the videos even if they provided robust evidence when interpreting students' understandings. In conclusion, the results obtained in individual video analysis confirmed the idea "deciding how to respond on the basis of children's understandings can occur only if teachers 
interpret children's understandings, and these interpretations can be made only if teachers attend to the details of children's strategies" (Jacobs et al. 2010, p. 197).

In the group discussions of micro-case videos, PMTs had opportunities to attend to and interpret students' understandings and decide how to respond on the basis of students' understanding again. In noticing studies, key indicators of advanced level noticing have been identified in many ways which are recalling specific mathematical details in students' understanding, supporting explanations with evidence, moving beyond description or evaluation, refraining from evaluating students' understanding in a dichotomy (right/wrong), and offering alternative interpretations and instructional actions on the basis of students' understandings (Callejo and Zapatera 2017; Jacobs et al. 2010; Krupa et al. 2017; SánchezMatamoros et al. 2019). In the current study, the results obtained in group discussions showed similar growth indicators in PMTs' professional noticing of students' understandings with the influences of their peers' ideas as follows: attending to specific mathematical elements in students' understandings, considering alternative interpretations of students' understandings and focusing on alternative instructional actions on the basis of the students' understandings.

In the literature, researchers mostly observed improvements in prospective teachers' skills to attend to and interpret student understanding. However, they generally found no or little change in the responding skill (Jacobs et al. 2010; Krupa et al. 2017; Schack et al. 2013). Differently, our study revealed that discussions of student mathematical understanding in micro-case videos after individual analysis of the videos affected the prospective teachers' responding skills positively. In particular, we found that the number and nature of instructional actions increased after the group discussions since $53 \%$ of the instructional actions were grouped into specific-detailed category although this percentage was only $13 \%$ in individual video analysis reflections. In these instructional actions, PMTs identified both mathematical elements and the cognitive mechanism involved in the understanding of trapezoids. In that process, we recognized that sharing knowledge in group discussions helped prospective teachers to develop, contrast and compare, and discuss different perspectives, arguments, and conjectures on the issue, and they reached a common understanding. As PMTs recognized different mathematical characteristics of students' understandings in group discussions, they concentrated on specific-detailed instructional actions. For example, studies about quadrilaterals suggest that if students distinguish examples (trapezoids) from non-examples (non-trapezoids), they will begin to develop a concept image of trapezoids (Popovic 2012). Alternatively, using a geometry software might be useful in supporting students' understanding of geometry because it allows dynamic manipulations to preserve the critical properties of a shape it contains (Erez and Yerushalmy 2006). In this study, similar specific-detailed instructional actions were mentioned by prospective teachers in the group discussions and post-discussion reflection papers.

To conclude, discussions of micro-case videos enabled prospective teachers to learn what and how the rest of group thought and gain different perspectives and alternative pedagogical ways on the basis of student mathematical understanding. Those results support several studies that emphasize the collaboration in promoting teacher noticing (e.g., van Es and Sherin 2002). From this point of view, we argue that careful production of micro-case videos, focusing on a specific mathematical topic, analyzing micro-case videos individually and discussing them with group members all contributed to prospective teachers' noticing of students' mathematical understanding. They are valuable indicators to introduce micro-case videos into teacher education programs in addition to classroom videos or written cases as a promising setting for the development of prospective teachers' noticing skills. Therefore, this study contributes to the field by providing an answer to the 
question of what should be done to increase the level of prospective teachers' professional noticing skills.

\section{Implications}

In light of the results obtained from our in-depth analysis, we want to share implications for future studies. In this study, we designed video segments that involved students' unexpected and inconsistent responses about trapezoids that prospective teachers could not anticipate in the pre-interviews. At this point, we think that micro-case videos provide a great contribution to mathematics educators to build upon research on teachers' professional noticing of students' understanding. Moreover, they help to adapt similar methodological ways in order to measure and support PMTs' noticing skills. In context-oriented noticing studies, prospective teachers either examine the students' written answers or directly watch the video clips that contain the students (e.g., Callejo and Zapatera 2017; Sánchez-Matamoros et al. 2019) or they discuss video clips (e.g., Krupa et al. 2017; Schack et al. 2013). In this study, we used a methodological way as follows: individual video analysis $\rightarrow$ writing a reflection paper $\rightarrow$ group discussion $\rightarrow$ writing a reflection paper. We recognized important growth indicators in prospective teachers' professional noticing skills when PMTs got opportunities to examine a video both individually and in a group. We think that using different methodological ways (e.g., working individually and in small groups and having large group discussions on students' works) might give the opportunity to researchers to contrast and compare the development of prospective teachers' professional noticing skills. Finally, we conducted pre-interviews to understand PMTs' knowledge of quadrilaterals for the production of micro-case videos instead of examining the relationship between teachers' knowledge and professional noticing skills. We think that this research can inspire the future research to examine these relationships.

\section{References}

Butuner, S. O., \& Filiz, M. (2017). Exploring Turkish mathematics teachers' content knowledge of quadrilaterals. International Journal of Research in Education and Science, 3(2), 395-408.

Callejo, M. L., \& Zapatera, A. (2017). Prospective primary teachers' noticing of students' understanding of pattern generalization. Journal of Mathematics Teacher Education, 20(4), 309-333.

Clement, J. (2000). Analysis of clinical interviews: Foundation and model viability. In R. Lesh \& A. E. Kelly (Eds.), Research design in mathematics and science education (pp. 547-589). Hillsdale: Erlbaum.

Colestock, A., \& Sherin, M. G. (2009). Teachers' sense-making strategies while watching video of mathematics instruction. Journal of Technology and Teacher Education, 17(1), 7-29.

Crespo, S. (2000). Seeing more than right and wrong answers: Prospective teachers' interpretations of students' mathematical work. Journal of Mathematics Teacher Education, 3(2), 155-181.

De Villiers, M. (1994). The role and function of a hierarchical classification of quadrilaterals. For the Learning of Mathematics, 14(1), 11-18.

Erez, M., \& Yerushalmy, M. (2006). If you can turn a rectangle into a square, you can turn a square into a rectangle: Young students' experience the dragging tool. International Journal of Computers for Mathematical Learning, 11(3), 271-299.

Fernández, C., Llinares, S., \& Valls, J. (2013). Primary school teacher's noticing of students' mathematical thinking in problem solving. The Mathematics Enthusiast, 10(1), 441-468.

Franke, M. L., Webb, N. M., Chan, A. G., Ing, M., Freund, D., \& Battey, D. (2009). Teacher questioning to elicit students' mathematical thinking in elementary school classrooms. Journal of Teacher Education, 60(4), 380-392. 
Freese, A. R. (2006). Reframing one's teaching: Discovering our teacher selves through reflection and inquiry. Teaching and Teacher Education, 22(1), 100-119.

Fujita, T. (2012). Learners' level of understanding of the inclusion relations of quadrilaterals and prototype phenomenon. The Journal of Mathematical Behavior, 31, 60-72.

Fujita, T., \& Jones, K. (2007). Learners' understanding of the definitions and hierarchical classification of quadrilaterals: Towards a theoretical framing. Research in Mathematics Education, 9(1\&2), 3-20.

Glogger-Frey, I., Deutscher, M., \& Renkl, A. (2018). Student teachers' prior knowledge as prerequisite to learn how to assess pupils' learning strategies. Teaching and Teacher Education, 76, 227-241. https:// doi.org/10.1016/j.tate.2018.01.012.

Hazzan, O., \& Zazkis, R. (1999). A Perspective on give an example tasks as opportunities to construct links among mathematical concepts. Focus on Learning Problems in Mathematics, 21(4), 1-14.

Jacobs, V. R., Franke, M. L., Carpenter, T. P., Levi, L., \& Battey, D. (2007). Professional development focused on children's algebraic reasoning in elementary school. Journal for Research in Mathematics Education, 48(3), 258-288.

Jacobs, V. R., Lamb, L. L. C., \& Philipp, R. A. (2010). Professional noticing of children's mathematical thinking. Journal for Research in Mathematics Education, 41(2), 169-202.

Jacobs, J. K., Yoshida, M., Fernández, C., \& Stigler, J. (1997). Japanese and American teachers' evaluations of mathematics lessons: A new technique for exploring beliefs. Journal of Mathematical Behavior, 16, 7-24.

Jaworski, B. (1990). Video as a tool for teachers' professional development. Professional Development in Education, 16(1), 60-65.

Josefsson, M. (2013). Characterizations of trapezoids. Forum Geometry, 13, 23-35.

Kang, H., \& van Es, E. A. (2019). Articulating design principles for productive use of video in preservice education. Journal of Teacher Education, 70(3), 237-250. https://doi.org/10.1177/0022487118 778549 .

Krupa, E. E., Huey, M., Lesseig, K., Casey, S., \& Monson, D. (2017). Investigating secondary preservice teacher noticing of students' mathematical thinking. In E. O. Schack, M. H. Fisher, \& J. A. Wilhelm (Eds.), Teacher noticing: Bridging and broadening perspectives, contexts, and frameworks (pp. 49-72). Cham: Springer.

Leatham, K. R., Peterson, B. E., Stockero, S. L., \& Van Zoest, L. R. (2015). Conceptualizing mathematically significant pedagogical opportunities to build on student thinking. Journal for Research in Mathematics Education, 46(1), 88-124.

Leung, I. K. C. (2008). Teaching and learning of inclusive and transitive properties among quadrilaterals by deductive reasoning with the aid of SmartBoard. ZDM Mathematics Education, 40, 1007-1021.

Mason, J. (2002). Researching your own practice: The discipline of noticing. New York: Routledge.

Mitchell, R. N., \& Marin, K. A. (2015). Examining the use of a structured analysis framework to support prospective teacher noticing. Journal of Mathematics Teacher Education, 18(6), 551-575.

Musburger, R. B., \& Ogden, M. R. (2014). Single-camera video production. Burlington, MA: Focal Press.

Okazaki, M., \& Fujita, T. (2007). Prototype phenomena and common cognitive paths in the understanding of the inclusion relations between quadrilaterals in Japan and Scotland. In Proceedings of the 31 st conference of the international group for the psychology of mathematics education (Vol. 4, pp. 41-48).

Peterson, B. E., \& Leatham, K. R. (2009). Learning to use students' mathematical thinking to orchestrate a class discussion. In L. Knott (Ed.), The role of mathematics discourse in producing leaders of discourse (pp. 99-128). Charlotte, NC: Information Age Publishing.

Popovic, G. (2012). Who is this trapezoid, anyway? Mathematics Teaching in the Middle School, 18(4), 196-199.

Sánchez-Matamoros, G., Fernández, C., \& Llinares, S. (2019). Relationships among prospective secondary mathematics teachers' skills of attending, interpreting and responding to students' understanding. Educational Studies in Mathematics, 100(1), 83-99.

Santagata, R., Zannoni, C., \& Stigler, J. W. (2007). The role of lesson analysis in pre-service teacher education: An empirical investigation of teacher learning from a virtual video-based field experience. Journal of Mathematics Teacher Education, 10(2), 123-140. https://doi.org/10.1007/s1085 7-007-9029-9.

Schack, E., Fisher, M., Thomas, J., Eisenhardt, S., Tassell, J., \& Yoder, M. (2013). Prospective elementary school teachers' professional noticing of children's early numeracy. Journal of Mathematics Teacher Education, 16, 379-397. https://doi.org/10.1007/s10857-013-9240-9. 
Seago, N. (2004). Using video as an object of inquiry for mathematics teaching and learning. In J. Brophy (Ed.), Advances in research on teaching: using video in teacher education (pp. 259-286). Amsterdam: Elsevier.

Seago, N., Koellner, K., \& Jacobs, J. (2018). Video in the middle: Purposeful design of video-based mathematics professional development. Contemporary Issues in Technology and Teacher Education, 18(1), 29-49.

Sherin, M. G. (2007). The development of teachers' professional vision in video clubs. In R. Goldman, R. D. Pea, B. Barron, \& S. J. Derry (Eds.), Video research in the learning sciences (pp. 383-395). Mahwah, NJ: Lawrence Erlbaum.

Sherin, M. G., Jacobs, V. R., \& Philipp, R. A. (2011). Mathematics teacher noticing: Seeing through teachers' eyes. New York: Routledge.

Sherin, M. G., Linsenmeier, K. A., \& van Es, E. A. (2009). Selecting video clips to promote mathematics teachers' discussion of student thinking. Journal of Teacher Education, 60(3), 213-230. https:// doi.org/10.1177/0022487109336967.

Sherin, M. G., \& van Es, E. A. (2009). Effects of video club participation on teachers' professional vision. Journal of Teacher Education, 60(1), 20-37. https://doi.org/10.1177/0022487108328155.

Son, J. (2013). How preservice teachers interpret and respond to student errors: Ratio and proportion in similar rectangles. Educational Studies in Mathematics, 84, 49-70.

Star, J. R., \& Strickland, S. K. (2008). Learning to observe: Using video to improve preservice mathematics teachers' ability to notice. Journal of Mathematics Teacher Education, 11, 107-125. https ://doi.org/10.1007/s10857-007-9063-7.

Stockero, S. L., Leatham, K. R., Van Zoest, L. R., \& Peterson, B. E. (2017a). Noticing distinctions among and within instances of student mathematical thinking. In E. O. Schack, M. H. Fisher, \& J. A. Wilhelm (Eds.), Teacher noticing: Bridging and broadening perspectives, contexts, and frameworks (pp. 467-480). Cham: Springer.

Stockero, S. L., Rupnow, R. L., \& Pascoe, A. E. (2017b). Learning to notice important student mathematical thinking in complex classroom interactions. Teaching and Teacher Education, 63, 384-395. https://doi.org/10.1016/j.tate.2017.01.006.

Stockero, S. L., \& Van Zoest, L. R. (2013). Characterizing pivotal teaching moments in beginning mathematics teachers' practice. Journal of Mathematics Teacher Education, 16(2), 125-147. https://doi. org/10.1007/s10857-012-9222-3.

Strauss, A. L., \& Corbin, J. M. (1998). Basis of qualitative research: Techniques and procedures for developing grounded theory. California: Sage Publications.

Superfine, A. C., Fisher, A., Bragelman, J., \& Amador, J. M. (2017). Shifting perspectives on preservice teachers' noticing of children's mathematical thinking. In E. O. Schack, M. H. Fisher, \& J. A. Wilhelm (Eds.), Teacher noticing: Bridging and broadening perspectives, contexts, and frameworks (pp. 409-426). Berlin: Springer. https://doi.org/10.1007/978-3-319-46753-5_24.

Superfine, A. C., Li, W., Bragelman, J., \& Fisher, A. (2015). Examining the use of video to support preservice elementary teachers' noticing of children's thinking. Journal of Technology and Teacher Education, 23(2), 137-157.

Talanquer, V., Bolger, M., \& Tomanek, D. (2015). Exploring prospective teachers' assessment practices: Noticing and interpreting student understanding in the assessment of written work. Journal of Research in Science Teaching, 52(5), 585-609. https://doi.org/10.1002/tea.21209.

Tall, D. O., \& Vinner, S. (1981). Concept image and concept definition in mathematics with special reference to limits and continuity. Educational Studies in Mathematics, 12(2), 151-169.

Türnüklü, E., Alaylı, F. G., \& Akkaş, E. N. (2013). Investigation of prospective primary mathematics teachers' perceptions and images for quadrilaterals. Educational Sciences: Theory \& Practice, 13(2), 1225-1232.

Ulusoy, F. (2015). A meta-classification for students' selections of quadrilaterals: The case of trapezoid. In K. Krainer \& N. Vondrová (Eds.), Proceedings of the ninth congress of the European society for research in mathematics education (CERME-9) (pp. 598-607). Prague: Faculty of Education and ERME, Charles University.

Ulusoy, F. (2016). The role of learners' example spaces in example generation and determination of two parallel and perpendicular line segments. In C. Csíkos, A. Rausch, \& J. Szitányi (Eds.), Proceedings of the 40th conference of the international group for the psychology of mathematics education (Vol. 4, pp. 299-306). Szeged: PME.

Ulusoy, F. (2020). Micro-case videos: A proposal for purposeful video design to enhance prospective teachers' noticing of student thinking. In J. Keengwe (Ed.), Handbook of research on innovative pedagogies and best practices in teacher education (pp. 310-331). Hershey, PA: IGI Global. https ://doi.org/10.4018/978-1-5225-9232-7.ch018. 
Ulusoy, F., \& Çakıroğlu, E. (2018). Using video cases and small-scale research projects to explore prospective mathematics teachers' noticing of student thinking. Eurasia Journal of Mathematics, Science and Technology Education, 14(9), em1571. https://doi.org/10.29333/ejmste/92020.

Usiskin, Z., \& Griffin, J. (2008). The classification of quadrilaterals: A study of definition., A Volume is Research in Mathematics Education Charlotte, NC: Information Age Publishing.

van Es, E. A. (2011). A framework for learning to notice student thinking. In M. G. Sherin, V. R. Jacobs, \& R. A. Philipp (Eds.), Mathematics teacher noticing: Seeing through teachers' eyes (pp. 134151). New York: Routledge.

van Es, E. A., Cashen, M., Barnhart, T., \& Auger, A. (2017). Learning to notice mathematics instruction: Using video to develop preservice teachers' vision of ambitious pedagogy. Cognition and Instruction, 35(3), 165-187.

van Es, E. A., \& Sherin, M. G. (2002). Learning to notice: Scaffolding new teachers' interpretations of classroom interactions. Journal of Technology and Teacher Education, 10(4), 571-596.

van Es, E. A., \& Sherin, M. G. (2008). Mathematics teachers' "learning to notice" in the context of a video club. Teaching and Teacher Education, 24(2), 244-276. https://doi.org/10.1016/j. tate.2006.11.00.

van Es, E. A., Tunney, J., Goldsmith, L. T., \& Seago, N. (2014). A framework for the facilitation of teachers' analysis of video. Journal of Teacher Education, 65(4), 340-356.

Vinner, S. (1991). The role of definitions in the teaching and learning of mathematics. In D. Tall (Ed.), Advanced mathematical thinking (pp. 65-81). Dordrecht: Kluwer.

Publisher's Note Springer Nature remains neutral with regard to jurisdictional claims in published maps and institutional affiliations. 